\title{
Use of Antibiotics during Immune Checkpoint Inhibitor Treatment Is Associated with Lower Survival in Hepatocellular Carcinoma
}

\author{
Ka Shing Cheung a, b Lok Ka Lam ${ }^{a}$ Wai Kay Seto ${ }^{a, b}$ Wai K. Leung ${ }^{a}$ \\ aDepartment of Medicine, The University of Hong Kong, Queen Mary Hospital, Hong Kong, China; ${ }^{\mathrm{b}}$ Department of \\ Medicine, The University of Hong Kong-Shenzhen Hospital, Shenzhen, China
}

\section{Keywords}

Hepatocellular carcinoma · Immunology · Liver cancer . Cirrhosis

\begin{abstract}
Background: Recent studies suggested that use of antibiotics may interfere with treatment responses to immune checkpoint inhibitors (ICls). We determined whether concurrent use of antibiotics during $\mathrm{ICl}$ therapy was associated with adverse outcomes in patients with advanced hepatocellular carcinoma (HCC). Methods: This is a territory-wide retrospective cohort study including all advanced HCC patients who received ICls (nivolumab, pembrolizumab, or ipilimumab) between January 2014 and December 2019. Exclusion criteria included prior liver transplantation and use of cabozantinib, regorafenib, or ramucirumab. The exposure of interest was concurrent antibiotic use within 30 days before or after the commencement of $\mathrm{ICI}$. The adjusted hazard ratio (aHR) of cancer-related mortality and all-cause mortality with antibiotic use was derived by propensity score (PS) matching in 1:2 ratio of covariates including baseline characteristics, causes of cirrhosis, Child-Pugh status, prior HCC treatment, comorbidities, concurrent medications, and laboratory results including alpha fetoprotein. Results: A total of 395 HCC patients who had received ICls were included. During a median follow-up of 16.5 months (interquartile
\end{abstract}

karger@karger.com www.karger.com/lic

Karger

bOPEN ACCESS
(C) 2021 The Author(s)

Published by S. Karger AG, Basel

This is an Open Access article licensed under the Creative Commons Attribution-NonCommercial-4.0 International License (CC BY-NC) (http://www.karger.com/Services/OpenAccessLicense), applicable to the online version of the article only. Usage and distribution for commercial purposes requires written permission. range [IQR]: 5.6-44.3), there were 286 (72.4\%) deaths including 231 cancer-related deaths. The median time from the first ICl to event was 7.7 months (IQR: 4.0-16.8). PS matching of 56 antibiotic users with 99 nonusers showed that concurrent antibiotic use with $\mathrm{ICl}$ was associated with higher cancer-related (aHR: 1.66; 95\% Cl: 1.08-2.54) and all-cause mortality (aHR: $1.63 ; 95 \% \mathrm{Cl}: 1.17-2.28)$. Conclusions: Concurrent antibiotic use during immunotherapy was associated with higher mortality in patients with advanced HCC. Further studies should examine the role of gut dysbiosis on responses to $\mathrm{ICl}$.

(c) 2021 The Author(s)

Published by S. Karger AG, Basel

\section{Background}

Liver cancer is the fourth leading cause of cancer-related mortality worldwide, with hepatocellular carcinoma (HCC) accounting for $75-85 \%$ of the cases [1]. The overall prognosis of HCC remains dismal as most patients are either diagnosed at advanced stage or with poor liver reserve due to the presence of liver cirrhosis, rendering curative therapy (resection or ablative therapy) or liver transplantation not feasible. The effectiveness of systemic therapy like sorafenib is suboptimal [2], and newer therapies including multi-kinase inhibitors (regorafenib and cabozantinib) and monoclonal antibody (ramuci- 
rumab) were also associated with a median overall survival of 8.5-10.6 months only [3].

Immune checkpoint inhibitors (ICIs) against antiprogrammed cell death 1 (anti-PD-1) or cytotoxic T-lymphocyte-associated protein 4 (CTLA-4) are new promising therapies that have been shown to prolong survival in patients with advanced HCC. In the CheckMate 040 trial, it was reported that nivolumab (anti-PD-1) had an objective response rate of $14 \%$, with duration of response of at least 12 months in 59\% and an overall median survival of 15.1 months [4]. Recently, combination therapy of nivolumab and ipilimumab was further shown to have manageable safety, with an even higher objective response rate of $32 \%$ and durable response leading to accelerated approval by the Food and Drug Administration of the USA [5].

Despite the promising results, not all patients with advanced HCC respond to ICIs. Attributable factors across tumor types of other organs suggest lack of pre-existing infiltrating lymphocytes [6] or functionally exhausted lymphocytes [7], low tumor mutational burden [8], as well as defective pathways involved in interferon-receptor signaling and in antigen presentation [9]. Notably, it has been reported that gut microbiota could affect the response to anti-PD-1 immunotherapy in patients with HCC, with higher taxa richness and more gene counts being noted in responders than those in nonresponders [10]. Prior retrospective analyses of clinical studies in other cancers including lung cancer [11], renal cell cancer $[11]$, and melanoma $[12,13]$ also demonstrated a lower clinical benefit of ICIs when patients were given antibiotics during ICI commencement. In a recent post hoc analysis of 2 randomized controlled trials of 757 patients receiving ICIs, use of antibiotics was associated with shorter overall survival in patients with non-small-cell lung cancer (NSCLC) [14].

However, studies investigating the impact of concurrent antibiotics on the outcomes of HCC patients receiving ICIs are lacking. Moreover, the nature of antibiotics has not been studied. Herein, we conducted a territorywide study from Hong Kong to determine whether the use of antibiotics was associated with adverse outcome in HCC patients receiving ICIs.

\section{Methods}

\section{Study Design and Data Source}

This was a retrospective cohort study by using data from the Clinical Data Analysis and Reporting System (CDARS), which is a territory-wide electronic health-care database managed by the

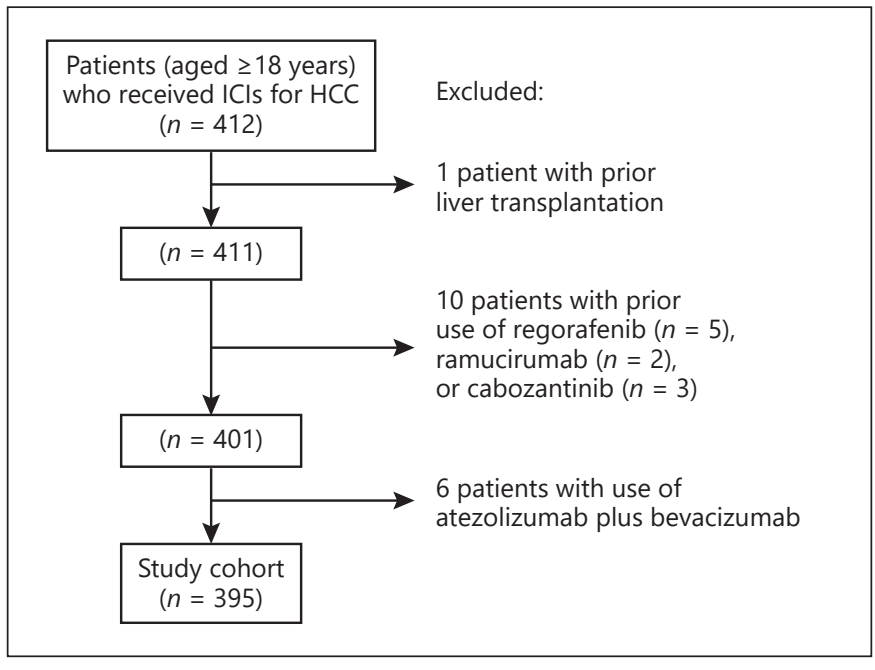

Fig. 1. Patient selection flow diagram. ICIs, immune checkpoint inhibitors; HCC, hepatocellular carcinoma.

Hong Kong Hospital Authority. The Hospital Authority is the only statutory public health-care body providing $90 \%$ of all primary, secondary, and tertiary medical care to the local population of $>7.5$ million. The CDARS contains data of patient's demographics, hospitalization, outpatient clinic visits, laboratory parameters, endoscopic and surgical procedures, as well as drug prescription and dispensing history. Diagnoses are represented by the codes of the International Classification of Diseases, Ninth Revision (ICD-9). The utilization of this electronic database enables researchers to publish territory-wide studies with a high degree of coding accuracy $(>90 \%)[15-17]$.

\section{Study Subjects}

Figure 1 illustrates the patient selection process. We identified all patients (aged 18 years or above) who had received ICIs (nivolumab, pembrolizumab, or ipilimumab) for advanced HCC between January 2014 and December 2019. HCC was identified by the diagnosis code of 155.0. Indications for patients to receive ICIs were due to ineligibility for curative procedures including surgery or ablative therapy and/or nonresponsiveness to tyrosine kinase inhibitors (sorafenib or lenvatinib) in the setting of clinical trials or out of label use. We excluded patients who had received atezolizumab plus bevacizumab, regorafenib, ramucirumab, or cabozantinib due to the small number of patients (atezolizumab plus bevaizumab: 6 , regorafenib: 5 , ramucirumab: 2 , and cabozantinib: 3 ) rendering propensity score (PS) matching between antibiotic users and nonusers not possible. In addition, patients with prior liver transplantation were excluded.

Validation of HCC Diagnosis Code

As individual's identification is anonymized in the electronic database, we could only verify the accuracy of diagnosis code of HCC in our center (Queen Mary Hospital), which is a tertiary referral center and a university teaching hospital. HCC was diagnosed by either histology or typical radiological features (arterial enhancement and venous wash-out by triphasic computed tomography scan or contrast magnetic resonance imaging). 


\section{Outcome Definition}

The primary outcome of interest was cancer-related mortality (with liver cancer specified as the cause of death in the database). The secondary outcome of interest was all-cause mortality. We observed patients from the start date of the first ICI (i.e., index date) till the occurrence of the outcome of interest or the end of study on June 30, 2020.

\section{Study Variables}

The exposure of interest was concurrent antibiotic use, defined as any antibiotic use within a period of 30 days either before or after the commencement of the first ICI. This interval was chosen in accordance with the previous studies $[11,14,18]$. Eleven classes of antibiotics were included as follows: penicillin, cephalosporins, macrolides, carbapenems, quinolones, tetracyclines, aminoglycosides, nitroimidazoles, glycopeptides, sulpha/trimethoprim, and others (nitrofurantoin, rifampicin, and rifaximin). None of these patients received monobactams, clindamycin, linezolid, and daptomycin. Online suppl. Table 1 (for all online suppl. material, see www.karger.com/doi/10.1159/000518090) shows the classification of antibiotics according to their effects on aerobes/anaerobes and spectrum of activity. Subgroup analysis was performed on the effects of nature of various antibiotics (anti-anaerobic vs. anti-aerobic and broad vs. narrow spectrum) on survival.

Other covariates included patient's characteristics, treatment status, concomitant drug usage, and laboratory parameters. Patient's characteristics were the age of receiving the first ICI, sex, causes of cirrhosis (chronic hepatitis B infection, chronic hepatitis $\mathrm{C}$ infection, alcoholic cirrhosis, or others), Child Pugh score, and comorbidities (alcohol-related diseases, diabetes mellitus, hypertension, ischemic heart disease, atrial fibrillation, congestive heart failure, and stroke). Treatment status included prior surgery (hepatectomy) or local ablative therapies (radiofrequency ablation, ethanol injection, and transarterial chemoembolization), type of ICIs and use of tyrosine kinase inhibitors (sorafenib or lenvatinib). Concomitant medications included prednisolone, aspirin [19], statins [20], proton pump inhibitors (PPIs) [14]. PPIs are potent suppressants of gastric acid production and are linked with gut dysbiosis and promotion of T-cell tolerance [18, 21, 22]. Online suppl. Table 2 shows the ICD-9 codes of the covariates.

Laboratory parameters included AFP [2], bilirubin, alkaline phosphatase, alanine aminotransferase, aspartate aminotransferase, albumin, globulin, international normalized ratio, platelet, creatinine, and sodium. Other laboratory parameters were chosen as they are components of either the Child Pugh score or model for end-stage liver disease-sodium score that reflect underlying liver function.

\section{Statistical Analysis}

All statistical analyses were performed by using the $\mathrm{R}$ version 3.2.3 (R Foundation for Statistical Computing) statistical software. Continuous variables were expressed as median and interquartile range (IQR). The Mann-Whitney U-test was used to compare continuous variables of 2 groups. The $\chi^{2}$ test or Fisher's exact test was applied for categorical variables. As absolute neutrophil count (ANC) [23], absolute lymphocyte count (ALC) [23], neutrophil-to-lymphocyte ratio (NLR) [24], platelet-lymphocyte ratio (PLR) [24], and lactate dehydrogenase (LDH) [25] were found to be associated with survival outcomes in the previous studies, we compared the baseline levels of these markers between antibiotic users and nonusers.
PS analysis was used to control for confounding due to potential selection bias in treatment allocation. The PS was estimated by multivariable logistic regression based on the aforementioned covariates. As such, any difference in risk of cancer-related and allcause mortality would be theoretically due to the effect of antibiotics. We used PS matching as the primary analysis to calculate the adjusted hazard ratio (aHR) of cancer-related and all-cause mortality with concurrent antibiotic use compared to nonuse. Antibiotic users were matched to nonusers in a 1:2 ratio without replacement using a greedy distance-based matching algorithm with the logit of the PS within 0.1 standard deviation. The balance of covariates between the 2 groups was assessed by absolute standardized difference (ASD), which was derived from the absolute difference in means or proportions divided by the pooled standard deviation. An ASD of $<0.20$ indicates good balance for a particular covariate. Imbalanced covariates with ASD $>0.20$ after PS matching were adjusted for in the regression model $[26,27]$. Sensitivity analyses were performed by PS regression adjustment and multivariable analysis. Further sensitivity analysis was performed by including NLR $\geq 5$ and PLR $\geq 200$ (with a prior study showing worsened survival [24]) into the multivariable Cox model.

The PS-adjusted absolute difference in risk of cancer-related and all-cause mortality between antibiotic users and nonusers was calculated as follows $(\mathrm{aHR}-1) \times($ crude incidence rate of mortality in antibiotic nonusers). The associations between cancer-related/ all-cause mortality and the nature of antibiotics (anti-anaerobic vs. anti-aerobic and broad vs. narrow spectrum) were also determined.

Another cohort of advanced HCC patients who were treated with sorafenib but not ICI between 2010 and 2019 was recruited for as control to determine whether the association between antibiotics and mortality was just a marker of disease complication. The aHR of cancer-related mortality with antibiotic use in sorafenib treated HCC patients was determined.

\section{Results}

\section{Patient's Characteristics and Data Validation}

Whole Cohort

Figure 1 shows the patient selection process. A total of 395 HCC patients were identified. There were 335 (84.8\%) male and the median age of receiving ICI was 61.0 years (IQR: 52.8-69.3). There were 109 (27.6\%) antibiotic users and $286(72.4 \%)$ antibiotic nonusers. The median duration of concurrent antibiotic use during ICI was 13 days (IQR: 8-22).

Four ICI regimens were used by these patients including 231 (58.5\%) nivolumab users, 100 (25.3\%) pembrolizumab users, and 64 (16.2\%) patients receiving combination therapy (either ipilimumab plus nivolumab $[n=26]$ or ipilimumab plus pembrolizumab $[n=38])$. Among these 395 patients, 275 (69.6\%) subjects were from our hospital, and 268 cases were confirmed to be HCC after review of records, yielding a positive predictive value of the ICD-9 diagnosis code of $97.5 \%$. 
Table 1. Characteristics of the study cohort after PS matching

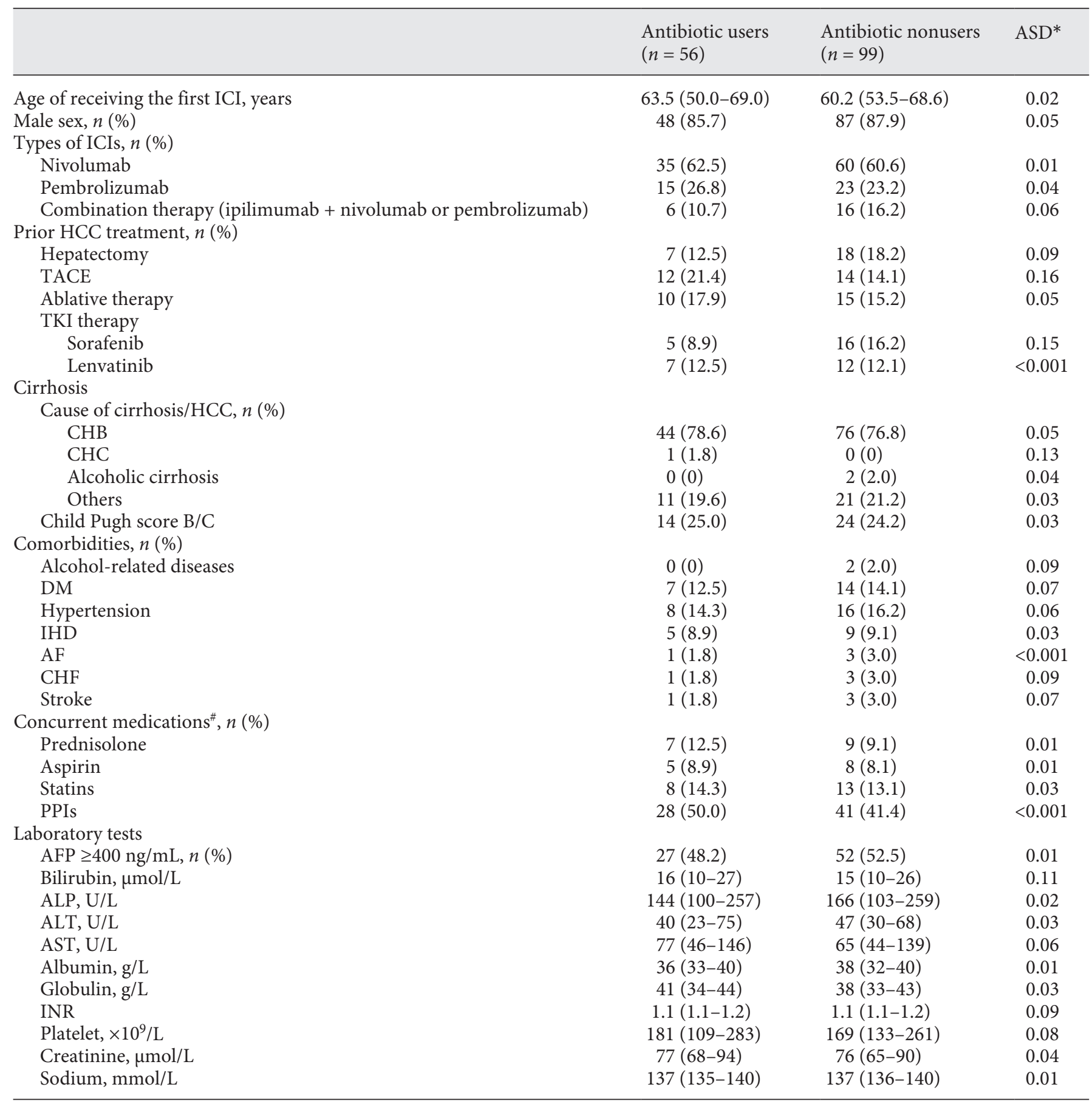

Continuous variables were expressed as median with IQR. Categorical variables were expressed as $n$ (\%). PS, propensity score; ASD, absolute standardized difference; ICI, immune checkpoint inhibitor; HCC, hepatocellular carcinoma; TACE, transarterial chemoembolization; TKI, tyrosine kinase inhibitor; CHB, chronic hepatitis B; CHC, chronic hepatitis C; DM, diabetes mellitus; IHD, ischemic heart disease; AF, atrial fibrillation; $\mathrm{CHF}$, congestive heart failure; PPIs, proton pump inhibitors; AFP, alpha fetoprotein; ALP, alkaline phosphatase; ALT, alanine aminotransferase; AST, aspartate aminotransferase; INR, international normalized ratio; IQR, interquartile range. * Variables with an ASD $>0.20$ is considered to be imbalanced. * Drug use was defined as any use within a window of 30 days before and 30 days after the start of the first ICI. 
Table 2. Association between antibiotics and mortality (cancer-related mortality and all-cause mortality) by various statistical analyses

\begin{tabular}{lcrr}
\hline & aHR & 95\% CI & $p$ value \\
\hline Cancer-related mortality & & & \\
$\quad$ PS matching $(n=155$, deaths $=96)$ & 1.66 & $1.08-2.54$ & 0.020 \\
$\quad$ PS regression adjustment $(n=395$, deaths $=231)$ & 1.55 & $1.07-2.26$ & 0.022 \\
$\quad$ Multivariable analysis $(n=395$, deaths $=231)$ & 1.74 & $1.17-2.57$ & 0.006 \\
All-cause mortality & & & \\
$\quad$ PS matching $(n=155$, deaths $=121)$ & 1.75 & $1.19-2.55$ & 0.004 \\
$\quad$ PS regression adjustment $(n=395$, deaths $=286)$ & 1.63 & $1.17-2.28$ & 0.004 \\
$\quad$ Multivariable analysis $(n=395$, deaths $=286)$ & 1.81 & $1.28-2.57$ & $<0.001$ \\
\hline
\end{tabular}

aHR, adjusted hazard ratio; 95\% CI, 95\% confidence interval; PS, propensity score; PPIs, proton pump inhibitors; TACE, transarterial chemoembolization; DM, diabetes mellitus; IHD, ischemic heart disease; AF, atrial fibrillation; CHF, congestive heart failure; AFP, alpha fetoprotein; ALP, alkaline phosphatase; ALT, alanine aminotransferase; AST, aspartate aminotransferase; INR, international normalized ratio. * Adjusted for age of receiving immunotherapy, sex, type of immunotherapy, prior hepatectomy/TACE/ablative therapy, alcohol-related diseases, DM, hypertension, IHD, AF, CHF, stroke, hepatic decompensation, cause of cirrhosis, prednisolone, aspirin, statins, PPIs, prior sorafenib/lenvatinib use, AFP, bilirubin, ALP, ALT, AST, albumin, globulin, INR, platelet, creatinine, sodium.

During a median follow-up of 16.5 months (IQR: 5.644.3 ) with 12,025 person-months, there were 286 (72.4\%) deaths including 231 cancer-related deaths. The causes of death were verified in the 268 HCC patients from our hospital. Among the antibiotic users, there were 98 (89.9\%) deaths including 78 (71.6\%) cancer-related deaths. For antibiotic nonusers, there were 188 (65.7\%) deaths including 153 (53.5\%) cancer-related deaths. The median time from receiving the first ICI to cancer-related and all-cause mortality was 7.7 months (IQR: 4.0-16.8) and 8.1 months (IQR: 4.3-20.4), respectively.

As the indications of antibiotics were not coded in the CDARS, we retrieved data of 76 antibiotic users from our own center. The most common indication for antibiotic use was fever of unknown cause or empirical use $(n=35)$, followed by pneumonia $(n=7)$, gastrointestinal bleeding $(n=5)$, and post-transarterial chemoembolization fever $(n=5)$. Other causes of antibiotic use were detailed in online suppl. Table 3.

The baseline characteristics of the 395 HCC patients before PS matching are shown in online suppl. Table 4 . There were a total of 109 (27.6\%) antibiotic users. Before PS matching, there was imbalance in some covariates (ASD >0.20) between antibiotic users and nonusers, including atrial fibrillation, causes of cirrhosis, certain laboratory parameters (bilirubin, alkaline phosphatase, aspartate aminotransferase, albumin, international normalized ratio, platelet, and sodium), and use of medications (prednisolone and PPIs).
PS-Matched Cohort

A total of 155 patients were included in the PS matching, including $56(36.1 \%)$ patients with concurrent antibiotic use and 99 nonusers. The median age was 61.0 years (IQR: 52.1-69.0), and there were 135 (87.1\%) males. All covariates were balanced between the 2 groups including age, sex, ICI therapies, Child-Pugh scores, comorbidities, concurrent medications, and laboratory parameters (Table 1).

\section{Association between Cancer-Related Mortality and Concurrent Antibiotic Use during ICIs}

After PS matching, concurrent antibiotic use with ICI was associated with a higher risk of cancer-related mortality (aHR: 1.66; 95\% CI: 1.08-2.54) (Table 2). Sensitivity analysis yielded consistent results (PS regression adjustment - aHR: 1.55, 95\% CI: 1.07-2.26; multivariable analysis - aHR: 1.74, 95\% CI: 1.17-2.57). The PS-adjusted absolute risk difference between antibiotic users and nonusers was 8.3 (95\% CI: 10.1-19.0) more cancer-related deaths per 1,000 person-months. For the control group of HCC patients treated with sorafenib but not ICI use $(n=$ 1,648 ; number of cancer-related deaths $=1,144$ ), the aHR of cancer-related mortality with antibiotic use was 1.10 (95\% CI: 0.97-1.26) on multivariable analysis.

\section{Association between All-Cause Mortality and \\ Concurrent Antibiotic Use during ICIs}

After PS matching, concurrent antibiotic use with ICI was also associated with a higher risk of all-cause mortal- 
Table 3. Association between antibiotics and mortality (cancer-related mortality and all-cause mortality) according to nature of antibiotics

\begin{tabular}{|c|c|c|c|}
\hline & $\mathrm{aHR}^{*}$ & $95 \% \mathrm{CI}$ & $p$ value \\
\hline \multicolumn{4}{|l|}{ Cancer-related mortality } \\
\hline \multicolumn{4}{|l|}{ Anti-anaerobic versus anti-aerobic activity } \\
\hline Nonuse $(n=286$, deaths $=153)$ & Ref & - & - \\
\hline Anti-anaerobic $(n=94$, deaths $=69)$ & 1.68 & $1.12-2.52$ & 0.013 \\
\hline Anti-aerobic $(n=15$, deaths $=9)$ & 2.24 & $1.04-4.83$ & 0.039 \\
\hline \multicolumn{4}{|l|}{ Duration of antibiotics } \\
\hline Nonuse $(n=286$, deaths $=153)$ & Ref & - & - \\
\hline$\leq 2$ weeks $(n=63$, deaths $=44)$ & 1.65 & $1.08-2.54$ & 0.021 \\
\hline$>2$ weeks $(n=46$, deaths $=34)$ & 1.94 & $1.14-3.30$ & 0.015 \\
\hline \multicolumn{4}{|l|}{ All-cause mortality } \\
\hline \multicolumn{4}{|l|}{ Anti-anaerobic versus anti-aerobic activity } \\
\hline Nonuse $(n=286$, deaths $=188)$ & Ref & - & - \\
\hline Anti-anaerobic $(n=94$, deaths $=86)$ & 1.77 & $1.23-2.54$ & 0.002 \\
\hline Anti-aerobic $(n=15$, deaths $=12)$ & 2.11 & $1.09-4.11$ & 0.027 \\
\hline \multicolumn{4}{|l|}{ Duration of antibiotics } \\
\hline Nonuse $(n=286$, deaths $=188)$ & Ref & - & - \\
\hline$\leq 2$ weeks $(n=63$, deaths $=56)$ & 1.77 & $1.21-2.60$ & 0.003 \\
\hline$>2$ weeks $(n=46$, deaths $=42)$ & 1.90 & $1.19-3.02$ & 0.007 \\
\hline
\end{tabular}

aHR, adjusted hazard ratio; 95\% CI, 95\% confidence interval; PPIs, proton pump inhibitors; TACE, transarterial chemoembolization; DM, diabetes mellitus; IHD, ischemic heart disease; AF, atrial fibrillation; CHF, congestive heart failure; AFP, alpha fetoprotein; ALP, alkaline phosphatase; ALT, alanine aminotransferase; AST, aspartate aminotransferase; INR, international normalized ratio. * Adjusted for age of receiving immunotherapy, sex, type of immunotherapy, prior hepatectomy/TACE/ablative therapy, alcoholrelated diseases, DM, hypertension, IHD, AF, CHF, stroke, hepatic decompensation, cause of cirrhosis, prednisolone, aspirin, statins, PPIs, prior sorafenib/lenvatinib use, AFP, bilirubin, ALP, ALT, AST, albumin, globulin, INR, platelet, creatinine, sodium. ity (aHR: 1.63; 95\% CI: 1.17-2.28) (Table 2). Sensitivity analysis yielded consistent results (PS regression adjustment - aHR: 1.75, 95\% CI: 1.19-2.55; multivariable analysis - aHR: 1.81, 95\% CI: 1.28-2.57). The PS-adjusted absolute risk difference between antibiotic users and nonusers was 11.7 (95\% CI: 3.2-23.7) more deaths per 1,000 person-months.

\section{Association between Mortality and Nature of Antibiotics}

Compared with antibiotic nonuse, both anti-anaerobic and anti-aerobic antibiotics were associated with higher cancer-related mortality (aHR: 1.68, 95\% CI: $1.12-$ 2.52; and aHR: $2.24,95 \%$ CI: $1.04-4.83$, respectively) as well as all-cause mortality (aHR: 1.77, 95\% CI: 1.23-2.54; and aHR: 2.11, 95\% CI: 1.09-4.11, respectively) (Table 3). All antibiotic users received at least 1 dose of broad-spectrum antibiotics, and therefore, analysis could not be performed for the effect of broad-spectrum versus narrowspectrum antibiotics on mortality.

Antibiotics and Immunotherapy on Survival of HCC Patients

\section{Association between Mortality and Duration of} Antibiotics

Compared with antibiotic nonuse, the aHR of cancerrelated mortality for those receiving antibiotics for $\leq 2$ weeks and $>2$ weeks was 1.65 (95\% CI: $1.08-2.54$ ) and 1.94 (95\% CI: 1.14-3.30), respectively. With regards to all-cause mortality, the aHR was 1.77 (95\% CI: 1.21-2.60) and 1.90 (95\% CI: 1.19-3.02) for those receiving antibiotics for $\leq 2$ weeks and $>2$ weeks, respectively (Table 3 ).

\section{Association between Mortality and Other Factors}

On multivariable analysis, apart from antibiotic usage, other factors associated with cancer-related mortality included male sex (aHR: 0.63, 95\% CI: 0.42-0.93), combination ICI therapy (ipilimumab plus nivolumab or pembrolizumab) (aHR: 0.56, 95\% CI: 0.38-0.82), prior sorafenib use (aHR: 1.70, 95\% CI: 1.10-2.60), cirrhosis, serum albumin (aHR: 0.96, 95\% CI: 0.93-0.99), and sodium level (aHR: 0.92, 95\% CI: 0.89-0.96). Similar association was observed for all-cause mortality (online suppl. Table 5). 
Comparison of ANC, ALC, NLR, PLR, and LDH

between Antibiotic Users and Nonusers

Missing data were noted in $2(0.5 \%)$ patients for peripheral neutrophil and lymphocyte counts and 221 patients (55.9\%) for $\mathrm{LDH}$. When comparing antibiotic users with nonusers, there were higher median ANC $\left(4.81 \times 10^{9} / \mathrm{L}\right.$ [IQR: $3.22-6.47$ ] vs. $3.77 \times 10^{9} / \mathrm{L}$ [IQR: $2.58-5.31$ ]; $p<$ 0.001 ), median NLR (5.15 [IQR: $3.43-7.81]$ vs. 3.41 [IQR: 2.25-5.86]; $p<0.001)$, median PLR (213 [IQR: 132-307] vs. 162 [IQR: 108-248]; $p<0.001)$, and median LDH (301 U/L [IQR: 221-433] vs. $252 \mathrm{U} / \mathrm{L}$ [IQR: 204-320]; $p=0.019$ ). However, there was no statistically significant difference in ALC $\left(0.94 \times 10^{9} / \mathrm{L}\right.$ [IQR: $0.67-1.33$ ] vs. $1.06 \times 10^{9} / \mathrm{L}$ [IQR: $0.75-1.40$ ]; $p=0.066)$. Sensitivity analysis was performed by including NLR and PLR into the multivariable Cox model. LDH was not included due to the significant proportion of missing data not favoring the use of multiple imputations. For cancer-related mortality, the aHR with antibiotic use was 1.64 (95\% CI: 1.10-2.45). For all-cause mortality, the aHR with antibiotic use was 1.75 (95\% CI: 1.23-2.49).

\section{Discussion}

While ICIs are increasingly used in patients with advanced HCC, this territory-wide cohort study showed that concurrent antibiotic use within 30 days of ICIs in HCC patients were associated with a $66 \%$ and $75 \%$ increase in cancer-related and all-cause mortality, respectively. The effect may appear higher for anti-aerobic than anti-anaerobic antibiotics.

To our knowledge, this is the first territory-wide study to demonstrate the potential harmful effects of antibiotics in HCC patients receiving ICIs. Our findings are consistent with prior studies reporting adverse effect of antibiotics in patients receiving ICIs for lung cancer, renal cell cancer, and melanoma [11-14]. A post hoc analysis of 757 NSCLC patients receiving atezolizumab reported that antibiotics were associated with $32 \%$ higher risk of mortality. Another study of 239 patients with lung cancer or renal cell cancer reported a 4.4 -fold increase in mortality with antibiotic use [11]. It was further shown that a more diverse gut microbiome was found in ICI responders than nonresponders [10-12], correlating with a larger number of T lymphocytes in the peripheral blood and tumor microenvironment. Antibiotics may therefore reduce ICI efficacy in HCC patients by lowering the gut microbial diversity with resultant change in the Tlymphocyte response.

Although prior studies had linked antibiotic use with adverse outcome in patients with other cancers, these studies did not look into the effect of nature of antibiotics. In this study, we showed that there was a higher risk of cancer-related death for antibiotics with anti-aerobic than anti-anaerobic activity (aHR: 2.24 vs. 1.68). A study of HCC patients receiving anti-PD-1 identified 20 responder-enriched species including Akkermansia muciniphila, Lachnospiraceae, and Ruminococcaceae which are anaerobes in preventing increase in intestinal permeability and systemic immunosuppression. On the other hand, the effect of antibiotic on the aerobes warrants more clinical studies.

Arguably, the observed effects of antibiotics may be confounded by disease severity, particularly the severity of underlying liver cirrhosis. However, this is unlikely to be of significant due to several reasons. First, the baseline characteristics (including liver function parameters, Child-Pugh score, comorbidities, and even AFP level) were well balanced $(\mathrm{ASD}<0.20)$ between antibiotics users and nonusers after PS matching (Table 1). Second, the recruitment of another control group of HCC patients with sorafenib but without ICI use shows that antibiotic use was not associated with a higher cancer-related mortality. This observation suggests that antibiotic use was not only a marker of disease complication that was associated with mortality but provides supporting evidence to the possible immunomodulatory role of antibiotics in ICIs. Third, the demonstration of harmful effects of antibiotics on cancer-related mortality rather than all-cause mortality as the latter could be confounded by the presence of severe infection. Last, we defined antibiotic exposure as use within a period of 30 days before or after the commencement of the first ICI $[11,14,18]$, and the median interval between the first ICI and cancer-related death, and all-cause mortality was 7.7 months and 8.1 months, respectively. This precludes the possibility of reverse causality in which severe infection preceding death mandates the use of antibiotics.

Severe limitations of the current study should be acknowledged. First, data on performance status such as the Eastern Cooperative Oncology Group performance status was not available in the electronic health database. However, there is unlikely a significant difference in Eastern Cooperative Oncology Group performance status between the 2 groups after extensive PS matching of a wide array of baseline characteristics. Second, imaging findings and hence disease status by using Response Evaluation Criteria in Solid Tumors were also not available on the CDARS, rendering evaluation of tumor progression not possible. However, immunotherapy was generally considered the last resort in HCC patients who had failed all con- 
ventional therapies during the study period and complete tumor response is unlikely, and hence, mortality is usually the best outcome measure in these patients. Third, inherent to all observational studies, there is a possibility of residual/unmeasured confounding despite PS matching of measured covariates. Fourth, the effect of individual antibiotic could not be ascertained due to underpower after multiple comparisons. Last, the biological mechanisms by which antibiotics impair ICI response could not be delineated in this retrospective cohort study. Future prospective cohorts with serial stool sample collection and microbiome profiling are needed to characterize the mechanistic pathways on how antibiotics impact the gut microbiome in HCC patients treated with ICIs.

\section{Conclusion}

In this territory-wide cohort study from Hong Kong, we showed that concurrent antibiotic use during ICI in HCC patients was associated with higher cancer-related and all-cause mortalities. Further studies should examine the role of gut dysbiosis on responses to ICIs in HCC patients.

\section{Statements of Ethics}

The study protocol conformed to the ethical guidelines of the World Medical Association Declaration of Helsinki and was approved by the Institutional Review Board of the University of
Hong Kong and the West Cluster of the Hong Kong Hospital Authority (reference No. UW 20-778). Written consent was exempt as this is a retrospective cohort study with no patient identification number involved.

\section{Conflict of Interest Statement}

K.S.C. has received speaker fee from AstraZeneca. W.K.L. has received speaker fee from Eisai, Ipsen and honorarium for attending advisory board for Janssen and Pfizer.

\section{Funding Sources}

This research received no external funding.

\section{Author Contributions}

Dr. Ka Shing Cheung was involved with study concept and design, literature search, analysis and interpretation of data, drafting of the manuscript, and approval of the final version of the manuscript. Dr. Lok Ka Lam was involved in data retrieval. Dr. Wai Kay Seto was involved in critical revision of the manuscript. Professor Wai K Leung was involved with the study concept and design, analysis and interpretation of data, critical revision of the manuscript for important intellectual content, study supervision, and approval of the final version of the manuscript.

\section{Data Availability Statement}

Datasets will be given upon reasonable request.

\section{References}

1 Bray F, Ferlay J, Soerjomataram I, Siegel RL, Torre LA, Jemal A. Global cancer statistics 2018: GLOBOCAN estimates of incidence and mortality worldwide for 36 cancers in 185 countries. CA Cancer J Clin. 2018;68(6):394424.

2 Lim H, Ramjeesingh R, Liu D, Tam VC, Knox JJ, Card PB, et al. Optimizing survival by changing the landscape of targeted therapy for intermediate and advanced hepatocellular carcinoma: a systematic review. J Natl Cancer Inst. 2020.

3 Ko KL, Mak LY, Cheung KS, Yuen MF. Hepatocellular carcinoma: recent advances and emerging medical therapies. F1000Res. 2020; 9:F1000.

4 El-Khoueiry AB, Sangro B, Yau T, Crocenzi TS, Kudo M, Hsu C, et al. Nivolumab in patients with advanced hepatocellular carcinoma (CheckMate 040): an open-label, noncomparative, phase $1 / 2$ dose escalation and expansion trial. Lancet. 2017;389(10088): 2492-502.

5 Yau T, Kang YK, Kim TY, El-Khoueiry AB, Santoro A, Sangro B, et al. Efficacy and safety of nivolumab plus ipilimumab in patients with advanced hepatocellular carcinoma previously treated with sorafenib: the checkmate 040 randomized clinical trial. JAMA Oncol. 2020.

6 Tumeh PC, Harview CL, Yearley JH, Shintaku IP, Taylor EJ, Robert L, et al. PD-1 blockade induces responses by inhibiting adaptive immune resistance. Nature. 2014;515(7528): 568-71.

7 Huang AC, Postow MA, Orlowski RJ, Mick R, Bengsch B, Manne S, et al. T-cell invigoration to tumour burden ratio associated with antiPD-1 response. Nature. 2017;545(7652):60-5.

8 Rizvi NA, Hellmann MD, Snyder A, Kvistborg P, Makarov V, Havel JJ, et al. Cancer immunology. Mutational landscape determines sensitivity to PD-1 blockade in non-small cell lung cancer. Science. 2015;348(6230):124-8.

9 Zaretsky JM, Garcia-Diaz A, Shin DS, EscuinOrdinas H, Hugo W, Hu-Lieskovan S, et al. Mutations associated with acquired resistance to PD-1 blockade in melanoma. N Engl J Med. 2016;375(9):819-29.

10 Zheng Y, Wang T, Tu X, Huang Y, Zhang H, Tan $\mathrm{D}$, et al. Gut microbiome affects the response to anti-PD-1 immunotherapy in patients with hepatocellular carcinoma. J Immunother Cancer. 2019;7(1):193.

11 Derosa L, Hellmann MD, Spaziano M, Halpenny D, Fidelle M, Rizvi H, et al. Negative association of antibiotics on clinical activity of immune checkpoint inhibitors in patients with advanced renal cell and non-small-cell lung cancer. Ann Oncol. 2018;29(6):1437-44.

12 Gopalakrishnan V, Spencer CN, Nezi L, Reuben A, Andrews MC, Karpinets TV, et al. Gut microbiome modulates response to anti- 
PD-1 immunotherapy in melanoma patients. Science. 2018;359(6371):97-103.

13 Matson V, Fessler J, Bao R, Chongsuwat T, Zha Y, Alegre ML, et al. The commensal microbiome is associated with anti-PD-1 efficacy in metastatic melanoma patients. Science. 2018;359(6371):104-8.

14 Chalabi M, Cardona A, Nagarkar DR, Dhawahir Scala A, Gandara DR, Rittmeyer A, et al. Efficacy of chemotherapy and atezolizum$\mathrm{ab}$ in patients with non-small-cell lung cancer receiving antibiotics and proton pump inhibitors: pooled post hoc analyses of the OAK and POPLAR trials. Ann Oncol. 2020;31(4): 525-31.

15 Cheung KS, Seto WK, Fung J, Mak LY, Lai CL, Yuen MF. Epidemiology and natural history of Wilson's disease in the Chinese: a territorybased study in Hong Kong between 2000 and 2016. World J Gastroenterol. 2017;23(43): 7716-26.

16 Cheung KS, Chen L, Chan EW, Seto WK, Wong ICK, Leung WK. Statins reduce the progression of non-advanced adenomas to colorectal cancer: a postcolonoscopy study in 187,897 patients. Gut. 2019;68(11):1979-85.
17 Cheung KS, Chan EW, Chen L, Seto WK, Wong ICK, Leung WK. Diabetes increases risk of gastric cancer after helicobacter pylori eradication: a territory-wide study with propensity score analysis. Diabetes Care. 2019; 42(9):1769-75.

18 Routy B, Le Chatelier E, Derosa L, Duong CPM, Alou MT, Daillère R, et al. Gut microbiome influences efficacy of PD-1-based immunotherapy against epithelial tumors. Science. 2018;359(6371):91-7.

19 Simon TG, Duberg AS, Aleman S, Chung RT, Chan AT, Ludvigsson JF. Association of aspirin with hepatocellular carcinoma and liver-related mortality. N Engl J Med. 2020;382(11):1018-28.

20 Lai SW. Statin therapy and mortality of hepatocellular carcinoma. Clin Gastroenterol Hepatol. 2020;18(1):265-6.

21 Imhann F, Bonder MJ, Vich Vila A, Fu J, Mujagic Z, Vork L, et al. Proton pump inhibitors affect the gut microbiome. Gut. 2016;65(5): 740-8.

22 Jackson MA, Goodrich JK, Maxan ME, Freedberg DE, Abrams JA, Poole AC, et al. Proton pump inhibitors alter the composition of the gut microbiota. Gut. 2016;65(5):749-56.
23 Zhang Y, Zeng G, Pan H, Li C, Hu Y, Chu K, et al. Safety, tolerability, and immunogenicity of an inactivated SARS-CoV-2 vaccine in healthy adults aged 18-59 years: a randomised, double-blind, placebo-controlled, phase 1/2 clinical trial. Lancet Infect Dis. 2021;21(2):181-92.

24 Russo A, Russano M, Franchina T, Migliorino MR, Aprile G, Mansueto G, et al. Neutrophilto-lymphocyte ratio (NLR), platelet-to-lymphocyte ratio (PLR), and outcomes with nivolumab in pretreated non-small cell lung cancer (NSCLC): a large retrospective multicenter study. Adv Ther. 2020;37(3):1145-55.

25 Shirotake S, Takamatsu K, Mizuno R, Kaneko GO, Nishimoto K, Oya M, et al. Serum lactate dehydrogenase before nivolumab treatment could be a therapeutic prognostic biomarker for patients with metastatic clear cell renal cell carcinoma. Anticancer Res. 2019;39(8):43717.

26 Austin PC. Balance diagnostics for comparing the distribution of baseline covariates between treatment groups in propensity-score matched samples. Stat Med. 2009;28(25): 3083-107.

27 Cheung KS, Leung WK, Seto WK. Application of big data analysis in gastrointestinal research. World J Gastroenterol. 2019;25(24): 2990-3008. 